\title{
summary
}

\section{Efficacy of electromyographic treatment is supported for temporomandibular disorders}

Crider AB, Glaros AG. A meta-analysis of EMG biofeedback treatment of temporomandibular disorders. J Orofacial Pain 1999; 13:29-37

Objective To determine the efficacy of electromyographic (EMG) biofeedback-based treatments and to estimate the size of treatment effect.

Data sources Medline (1966-1995), Psychlit (1990-1997), review articles and the authors' files.

Study selection Thirteen studies were identified, six of which were controlled trials, four were comparative trials and three were uncontrolled trials.

Results Data from 12 studies contributed to a meta-analysis that compared the size of pre- and post-treatment effect for EMG biofeedback with the size of effect for control conditions. Mean effect sizes for both reported pain and clinical examination outcomes were substantially larger for biofeedback treatments than for control conditions. In addition, $69 \%$ of patients who received EMG biofeedback treatments were rated as symptom-free or significantly improved, compared with only $35 \%$ of patients who were treated with a variety of placebo interventions only.

Conclusion Although limited in extent, the available data support the efficacy of EMG biofeedback treatments for temporomandibular disorders (TMD).

Address for reprints: Dr Alan G Glaros, 650 East 25th Street, Kansas City MI 64108, USA. Fax: +1 816235 2157. E-mail: glarosa@umkc.edu

\section{Commentary}

This comprehensive review assesses the outcome of six to 12 sessions of EMG biofeedback from the masseter muscle alone or in combination with stress management for TMD. Three outcome-categories (global improvements, reported pain and clinical examinations) were evaluated in patients of typical age- and sex-distributions for TMD. More than half of the patients had failed to improve with previous conservative treatment. Substantial improvements, particularly in pain, were to be found about twice as often as in placebo or in no-treatment control groups, and these improvements remained at long-term followup. The variation in improvement was great, although treatment results are in line with those of several other conservative interventions for TMD.

As the authors point out, TMD embraces a heterogeneous group of clinical problems and the etiology is not well understood. The analysis includes 2 decades of research and the diagnostic concepts have varied over time. Criteria for inclusion or exclusion in the studies have also varied, therefore. Little is known about what characteristics make patients more suitable for the treamtent method.

In general, the intervention aims at reducing muscular activity in frequency, intensity and/or duration, in individuals who have myofacial pain. The significance of the commonlyembraced etiological concept of muscular hyperactivity has been questioned. An attractive quality with EMG biofeedback is that it objectively measures the variable that it is supposed to change. Although a reduced EMG activity can be observed within sessions, no study has shown a reduction over time. Moreover, subjective or clinical pre- to post-treatment improvements have not correlated well with EMG data. Alternative hypotheses have therefore been advanced and are discussed thoroughly by the authors. One theory suggests that biofeedback enhances awareness of muscular activity in general and thereby leads to increased control and less parafunctional activity, which is possible but not proved. Another suggestion is that the patients perceptions of control, not their actual abilities to control, are altered and result in improved coping and selfefficacy. Cognitive changes are thus also a possibility.

The superiority of any particular conservative treatment approach over another in TMD has seldom been proved. Biofeedback is a reversible and safe method, in line with many treatment recommendations for TMD, but is it efficient? Even if the authors' conclusions about positive treatment efficacy are correct, the mechanisms behind them remain unexplained.

Lars Dahlström Sahlgrenska University Hospital, Mölndal, Sweden 